\title{
Identification fonctionnelle du canal calcique du réticulum sarcoplasmique
}

La contraction des muscles cardiaque et squelettique est déclenchée à la suite de la dépolarisation de la membrane de surface de leurs cellules et de la libération rapide du calcium contenu dans le réticulum sarcoplasmique (RS). Des mesures électrophysiologiques réalisées lors d'expériences de reconstitution membranaire ont montré qu'un canal $\mathrm{Ca}^{2+}$ sélectif est présent dans la membrane des microvésicules dérivées du réticulum sarcoplasmique. Son activité est modulée par le $\mathrm{Ca}^{2+}$ et l'ATP, qui augmentent sa probabilité d'ouverture, alors que le $\mathrm{Mg}^{2+}$ et la calmoduline ont des effets inhibiteurs. La ryanodine, un alcaloïde qui modifie le fonctionnement de ce canal de façon très spécifique, a servi d'outil pharmacologique pour identifier et purifier le complexe protéique qui forme à la fois le canal calcique du réticulum sarcoplasmique et la structure physique qui fait le lien entre cet organite et la membrane cellulaire. D'un point de vue clinique, un défaut génétique dans l'expression de cette protéine au niveau des muscles squelettiques est impliqué dans certaines formes du syndrome rare, mais létal, de l'hyperthermie maligne.

\section{Éric Rousseau} Hélène Chabot

\section{ADRESSE ET TIRÉS À PART}

Éric Rousseau : professeur adjoint. Hélène Chabot : étudiante graduée. Département de physiologie et biophysique, faculté de médecine, université de Sherbrooke, Sherbrooke

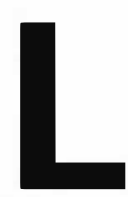

e travail mécanique produit par les cellules musculaires, squelettiques et cardiaques (figure 1), résulte des interactions chimiques entre les filaments d'actine et de myosine qui composent les unités contractiles appelées sarcomères. Cette activité mécanique est déclenchée par une augmentation transitoire du calcium cytoplasmique [1]. Ces variations rapides de la concentration de $\mathrm{Ca}^{2+}$ intracellulaire se produisent à la suite de la dépolarisation électrique (potentiel d'action) de la membrane de surface et du système tubulaire trans- verse (ou système $T$ ). C'est au moment où le signal électrique atteint le système $T$ qu'il induit une libération importante et rapide du $\mathrm{Ca}^{2+}$ emmagasiné dans le réticulum sarcoplasmique (RS). La nature exacte du signal transmis du système $\mathrm{T}$ au RS et les mécanismes précis qui contrôlent la libération du $\mathrm{Ca}^{2+}$ contenu dans ce dernier restent deux questions majeures de la physiologie musculaire puisqu'elles constituent des chaînons manquants du couplage excitation-contraction [1].

Parmi les mécanismes proposés, deux hypothèses plausibles sont actuelle 
ment retenues: (1) une dépolarisation de la membrane du système $T$ entraînerait une entrée de $\mathrm{Ca}^{2}+$ à travers les canaux sélectifs de cet ion. $\mathrm{Ce} \mathrm{Ca}^{2+}$ induirait à son tour une libération rapide et massive du $\mathrm{Ca}^{2+}$ contenu dans le réticulum sarcoplasmique, phénomène connu sous le nom de $\mathrm{Ca}^{2+}$ induced-Ca ${ }^{2+}$ release [2] qui constitue l'hypothèse du couplage ionique ; (2) des résultats expérimentaux récents démontrent que, lors des variations de champ électrique qui accompagnent la dépolarisation de la membrane du système $T$, des struc- tures protéiques spécialisées subissent un changement de conformation qui contrôlerait la libération de $\mathrm{Ca}^{2+}$ en modulant la perméabilité de la membrane du réticulum sarcoplasmique à ce cation divalent [3].

L'une des stratégies adoptées pour identifier les structures et les facteurs susceptibles de contrôler les mouvements de $\mathrm{Ca}^{2+}$ au niveau du réticulum sarcoplasmique a consisté à isoler les fractions microsomales dérivées de cet organite [4]. Les fractions enrichies en membrane du réticulum sarcoplasmique ont initialement permis

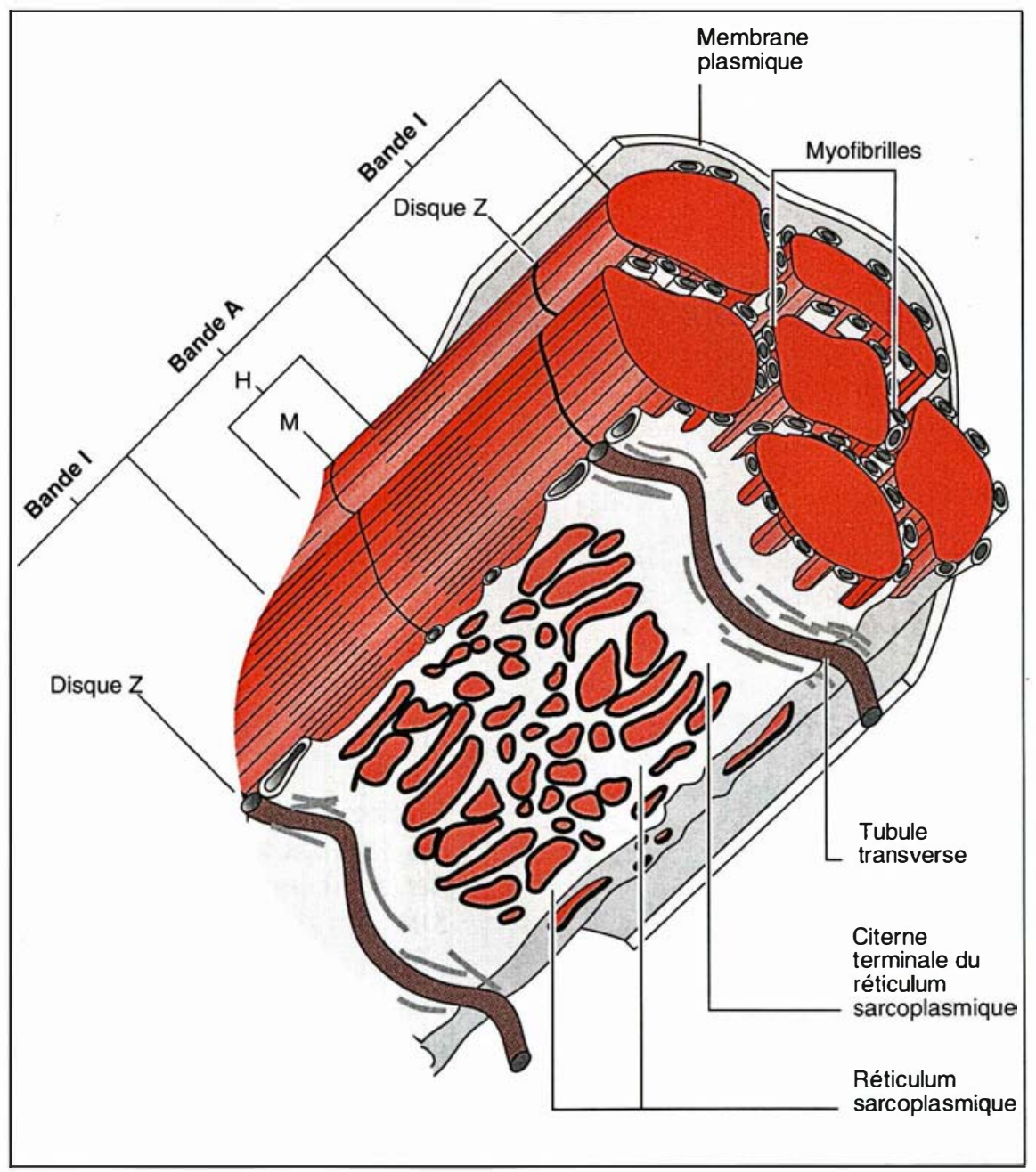

Figure 1. Structure tridimensionnelle d'une fibre musculaire. Les tubules transverses représentent des invaginations de la membrane plasmique qui sont en contiguïté avec les citernes terminales du réticulum sarcoplasmique. II a été postulé, puis démontré que le couplage entre l'excitation et la contraction $s^{\prime}$ effectue dans ces zones jonctionnelles qui correspondent aux disques $z$ de myofibrilles. Bandes $I$ : bandes constituées de filaments fins $d$ 'actine. Bandes $A$ : zones de juxtaposition entre les myofibrilles. Disque $Z$ : zone d'attachement des filaments d'actine. d'étudier les propriétés et le mode de fonctionnement de la pompe au $\mathrm{Ca}^{2+}\left(\mathrm{Ca}^{2+}-\mathrm{ATPase}\right.$ dépendante du $\mathrm{Mg}^{2+}$ ) qui permet le remplissage du réticulum sarcoplasmique [1]. Plus récemment [5], ces fractions microsomales ont été utilisées pour étudier les propriétés biochimiques des structures contrôlant le passage passif des ions à travers la membrane du réticulum sarcoplasmique (figure 2, p. 953).

Le but de cet article est de présenter les progrès réalisés ces dernières années quant à l'identification des protéines et des substances impliquées dans le contrôle de la libération du $\mathrm{Ca}^{2+}$ au niveau du réticulum sarcoplasmique. L'inaccessibilité et la petite taille du réticulum sarcoplasmique ont orienté les recherches vers des expériences de reconstitution membranaire $[6,7]$. Cette approche, qui consiste à faire fusionner des microvésicules du réticulum sarcoplasmique dans des bicouches lipidiques planes (BLP), permet d'étudier au niveau moléculaire les caractéristiques du canal calcique du réticulum sarcoplasmique des muscles squelettique [7] et cardiaque [8]. Elle a également permis de démontrer la sélectivité de ce canal aux cations divalents et sa sensibilité à différents agents connus pour moduler la perméabilité calcique du réticulum sarcoplasmique $[9,10]$. La purification et la reconstitution du récepteur de la ryanodine dans des membranes artificielles a prouvé que ce complexe protéique formait à la fois le canal calcique du réticulum sarcoplasmique et les piliers qui relient la membrane du réticulum sarcoplasmique à celle du système tubulaire transverse [11].

\section{Structure et rôle du réticulum sarcoplasmique}

Le réticulum sarcoplasmique constitue un réseau de membranes intracellulaires intercalées entre les myofilaments. Les études ultrastructurales ont montré que le réticulum sarcoplasmique est composé de deux régions : le réticulum sarcoplasmique longitudinal est disposé entre les sarcomères parallèlement à l'axe des cellules musculaires alors que le réticulum sarcoplasmique jonctionnel correspond aux extrémités du réseau qui forme des renflements, appelés citer- 


\section{RÉFÉRENCES}

1. Martonosi A. Mechanisms of $\mathrm{Ca}^{2+}$ release from sarcoplasmic reticulum of skeletal muscle. Physiol Rev 1984; 64: 1240-320.

2. Fabiato A. Myoplasmic free calcium concentration reached during the twitch of an intact isolated cardiac cell and during calcium induced release of calcium from the sarcoplasmic reticulum of a skinned cardiac cell from the adult rat or rabbit ventricle. $J$ Gen Physiol 1981 ; 78 : 457-97.

3. Brum G, Fitts R, Pizarro G, Rios E. A mobile charge of the skeletal muscle membrane requires calcium channel to function as voltage sensor of excitation contraction coupling. J Physiol (Lond) 1987 ; 398 : 475-505.

4. Meissner G. Adenine nucleotide stimulation of $\mathrm{Ca}^{2+}$ - induced $\mathrm{Ca}^{2+}$ release in sarcoplasmic reticulum. J Biol Chem 1984 ; 259:2365-74.

5. Meissner G, Darling E, Eveleth J. Kinetics of rapid $\mathrm{Ca}^{2}+$ release by sarcoplasmic reticulum : effects of $\mathrm{Ca}^{2+}, \mathrm{Mg}^{2+}$ and adenine nucleotides. Biochemistry $1986 ; 25$ : 236-44.

6. Smith JS, Coronado R, Meissner G. Sarcoplasmic reticulum contains adenine nucleotide activated calcium channels. Nature $1985 ; 316$ : 446-9.

7. Smith JS, Coronado R, Meissner G. Single channel measurements of the calcium release channel from skeletal muscle sarcoplasmic reticulum: activation by $\mathrm{Ca}^{2+}$ ATP and modulation by $\mathrm{Mg}^{2+}$. J Gen Physiol 1986 ; 88 : 573-88.

8. Rousseau E, Smith JS, Henderson JS, Meissner G. Single channel and ${ }^{45} \mathrm{Ca}^{2+}$ flux measurements of the cardiac sarcoplasmic reticulum calcium channel. Biophys $J$ $1986 ; 50: 1009-14$

9. Rousseau E, Ladine J, Liu QY, Meissner $\mathrm{G}$. Activation of the $\mathrm{Ca}^{2}+$ release channel of skeletal muscle sarcoplasmic reticulum by caffeine and related compounds. Arch Biochem Biophys 1988 ; 267 : 75-86.

10. Rousseau E, Smith JS, Meissner G. Ryanodine modifies conductances and gating behavior of single $\mathrm{Ca}^{2}+$ release channel. Am J Physiol 1987 ; 253 : C364-8.

11. Lai A, Erickson HP, Rousseau E, Liu QY, Meissner G. Purification and reconstitution of the calcium release channel from skeletal muscle. Nature 1988 ; 331 : 315-9.

12. Sommer JR, Johnson EA. Handbook of Physiology. In : Berne RM, ed. American Physiological Society Section, 2nd ed. Bethesda nes terminales. Ces dernières semblent être apposées à la membrane du système $\mathrm{T}$ des muscles squelettiques et à la membrane de surface dans le cas des cellules cardiaques [12].

Le rôle du réticulum sarcoplasmique, qui est de contrôler les niveaux de calcium intracellulaire, peut être corrélé à l'aptitude des fractions vésiculaires, enrichies en membranes dérivées de cet organite, à transporter le calcium $[13,14]$.

L'utilisation de ces préparations a permis d'étudier en détail les propriétés biochimiques de la pompe calcique, la $\mathrm{Ca}^{2+}$-ATPase-dépendante du $\mathrm{Mg}^{2+}$ [15]. D'ailleurs, cette enzyme recouvre littéralement le réticulum sarcoplasmique puisqu'il représente 60 à $85 \%$ des protéines de la membrane du réticulum sarcoplasmique des muscles striés. En outre, il est intéressant de constater que les microvésicules dérivées du réticulum

\section{* GLOSSAIRE}

AMP-PCP : analogue chimique non hydrolysable de l'adénosine triphosphate.

BLP : bicouche lipidique plane.

CHAPS : 3-[(3cholamidopropyl) diméthyl ammonio]-1 propanesulfonate, détergent doux.

Hyperthermie maligne: maladie héréditaire létale caractérisée par une sensibilité anormale aux anesthésiques volatils causant une contraction généralisée et une élévation irréversible de la température corporelle.

\section{IP3 : inositol triphosphate.}

$\boldsymbol{P C R}$ : polymerase-chain reaction, technique permettant l'amplification spécifique de fragments $d^{\prime} A D N$ à l'aide d'une polymérase.

pS : pico siemens, unité de conductance, équivalente à $10^{-12}$ mho.

Ryanodine : alcaloïde isolé de Ryania speciosa.

Sarcomère : espace des myofilaments séparant deux stries $Z$ adjacentes. sarcoplasmique jonctionnel sont plus denses car elles contiennent des inclusions protéiques telles que la calséquestrine, une molécule de $60 \mathrm{kDa}$ qui lie le $\mathrm{Ca}^{2+}$, et des protubérances protéiques caractéristiques qui ont été identifiées comme étant les piliers (feet structure) qui relient la membrane du réticulum sarcoplasmique au système T [16]. Sur le plan fonctionnel, les différentes fractions se différencient par leur aptitude à libérer leur $\mathrm{Ca}^{2+}$ intravésiculaire. Les vésicules dérivées du réticulum sarcoplasmique jonctionnel présentent un flux sortant calcique beaucoup plus rapide que celui mesuré sur les fractions enrichies en réticulum sarcoplasmique longitudinal. Cette différence de perméabilité du $\mathrm{Ca}^{2+}$ semble indiquer une distribution non uniforme des canaux ioniques, sélectif au $\mathrm{Ca}^{2+}$ à la surface du réticulum sarcoplasmique [5-9]. L'ensemble de ces observations suggère que les mouvements de $\mathrm{Ca}^{2+}$ à travers la membrane du réticulum sarcoplasmique sont sous le contrôle de mécanismes précis de régulation et qu'ils seraient localisés de façon préférentielle dans le réticulum sarcoplasmique jonctionnel. En revanche, les diverses fractions issues du réticulum sarcoplasmique présentent toutes une grande perméabilité aux ions monovalents $\left(\mathrm{K}^{+}, \mathrm{Cl}^{-}\right)$. Ces perméabilités ioniques serviraient de contre-transport de charges permettant de maintenir l'électroneutralité du système lors de la libération et du repompage du $\mathrm{Ca}^{2+}$ (figures $1 B$ et 2A).

\section{Pharmacologie de la libération du $\mathrm{Ca}^{2+}$ du réticulum sarcoplasmique}

Différentes approches expérimentales ont permis d'obtenir des informations précises sur les mécanismes moléculaires qui contrôlent les flux de $\mathrm{Ca}^{2+}$ à travers la membrane du réticulum sarcoplasmique. Les mesures de flux isotopiques ont montré que la libération du ${ }^{45} \mathrm{Ca}^{2+}$ intravésiculaire est modulée par le $\mathrm{Ca}^{2+}$ libre extravésiculaire $[5,8]$. Le flux sortant de ${ }^{45} \mathrm{Ca}^{2+}$ est maximal en présence de concentrations micromolaires de $\mathrm{Ca}^{2+}$ extravésiculaire. Il est minimal en présence de bloqueurs 


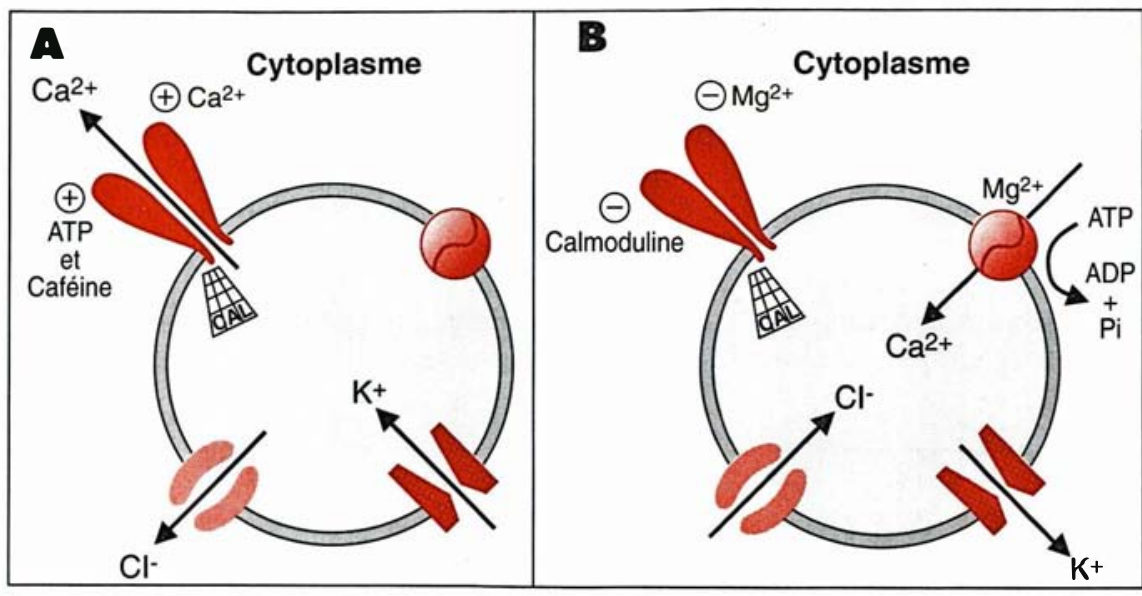

Figure 2. Représentation schématique des mouvements des ions à travers la membrane du réticulum sarcoplasmique. A. La libération rapide de $\mathrm{Ca}^{2+}$ qui est déclenchée par différents agents est un phénomène passif qui s'effectue selon le gradient de concentration. Elle est accompagnée de mouvements $d^{\prime}$ autres ions. Les anions $(\mathrm{Cl}-)$ ont tendance à quitter la lumière du réticulum sarcoplasmique alors que les cations monovalents $\left(K^{+}\right)$se déplacent dans le sens opposé afin de maintenir l'électroneutralité du système. B. Le repompage du $\mathrm{Ca}^{2+}$ dans le réticulum sarcoplasmique est un phénomène actif qui permet à la fois de contrôler le niveau de $\mathrm{Ca}^{2}+$ cytoplasmique et de rétablir le gradient de concentration calcique. Dans le réticulum sarcoplasmique, une grande partie du $\mathrm{Ca}^{2+}$ est liée à la calséquestrine (CAL).

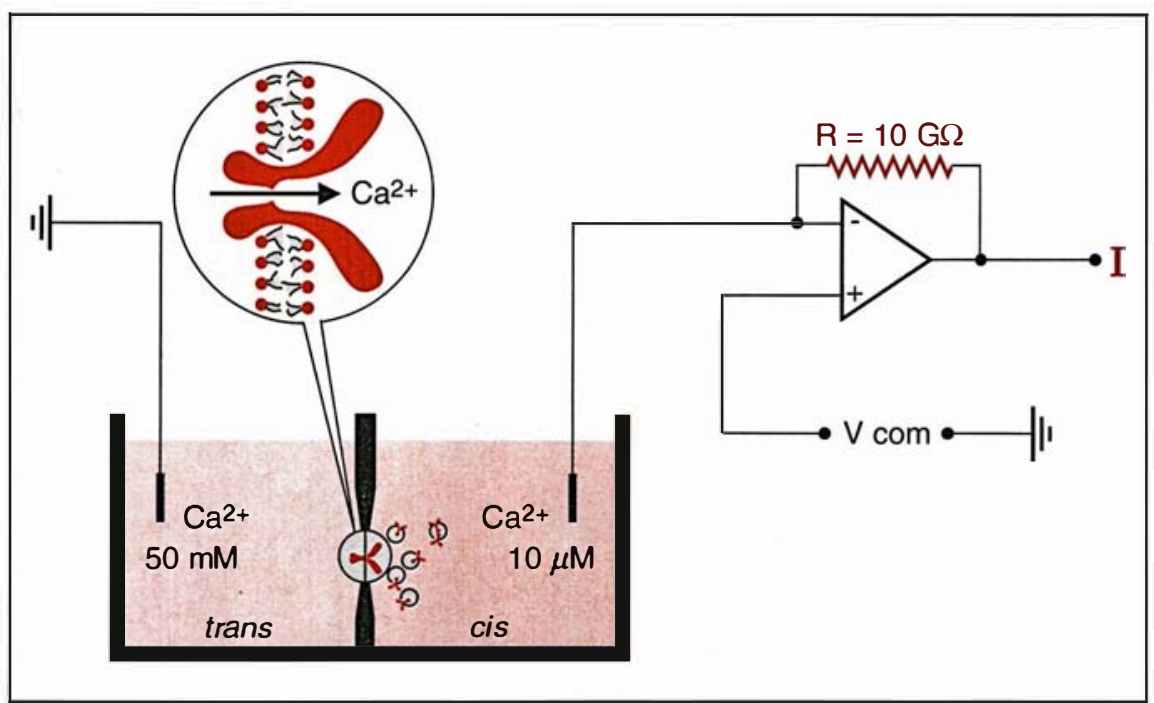

Figure 3. Schéma du montage et des conditions expérimentales utilisées pour étudier le mode de fonctionnement des canaux du réticulum sarcoplasmique. Les deux chambres expérimentales cis et trans contiennent des solutions salines de composition connues. Elles sont séparées par un septum perforé d'un orifice de $200 \mu \mathrm{m}$ de diamètre dans lequel une bicouche lipidique plane est formée à l'aide d'un mélange de phospholipides. Chaque chambre est reliée par une électrode au montage électronique qui permet de fixer le voltage appliqué (Vcom) et de mesurer le courant ionique (I) passant à travers la (ou les) protéine(s) incorporée(s) dans la membrane artificielle suite à la fusion des microvésicules. Les effets d'agents biochimiques ou pharmacologiques peuvent être testés de part et d'autre du canal reconstitué. Cette approche représente la seule possibilité pour étudier les canaux ioniques des structures non accessibles par la technique de patch clamp [36].

$\mathrm{m} / \mathrm{s} n^{\circ} 9$ vol. 8 , novembre 92 entre le $\mathrm{Mg}^{2+}$ et le rouge de ruthénium ou d'une concentration de $\mathrm{Ca}^{2+}$ extravésiculaire inférieure ou égale à $0,1 \mu \mathrm{M}$. L'addition d'ATP ou d'AMP-PCP potentialisent l'effet dépendant du $\mathrm{Ca}^{2+}$. La sensibilité de ce mécanisme à des ligands physiologiques tels que le $\mathrm{Ca}^{2+}$, les adénine-nucléotides, le $\mathrm{Mg}^{2+}$ ou la calmoduline constitue la preuve biochimique de l'existence d'une structure spécifique qui contrôle la perméabilité de la membrane du réticulum sarcoplasmique au $\mathrm{Ca}^{2+}$. Des mesures optiques, utilisant des sondes fluorescentes sensibles aux variations de $\mathrm{Ca}^{2+}$, ont confirmé que la libération de $\mathrm{Ca}^{2+}$ pouvait être déclenchée par des ligands physiologiques $\left(\mathrm{Ca}^{2+}, \mathrm{ATP}\right)$ ou par des substances aussi différentes que la caféine, l'halothane (un agent anesthésique) ou la doxorubicine (une substance anticancéreuse) [17]. La ryanodine, une substance naturelle qui a longtemps été utilisée comme insecticide, est connue pour modifier le couplage excitation-contraction des muscles striés. Des expériences électrophysiologiques ont démontré que la ryanodine n'affectait ni les phénomènes électriques de la membrane de surface ni l'activité de la pompe au $\mathrm{Ca}^{2+} \mathrm{du}$ réticulum sarcoplasmique, mais qu'elle interfere spécifiquement avec le processus de libération du calcium [19].

\section{Contrôle du canal calcique du réticulum sarcoplasmique}

Dans le but de visualiser et d'étudier l'activité des canaux ioniques du réticulum sarcoplasmique, les microvésicules ont été fusionnées dans des bicouches lipidiques planes en utilisant le montage décrit sur la figure 3 . Dans ces conditions expérimentales, un canal de grande conductance sélectif des cations divalents fut mis en évidence dans la membrane du réticulum sarcoplasmique de muscle squelettique [6]. Une structure similaire fut par la suite décrite au niveau du réticulum sarcoplasmique cardiaque [8]. La probabilité d'ouverture de ce canal augmente quand la concentration de $\mathrm{Ca}^{2+}$ passe de 0,1 à $10 \mu \mathrm{M}$ (figures $4 A$ et $4 B, \quad$ p. 954). Cette activation dépendante du $\mathrm{Ca}^{2+}$ est potentialisée en présence 


\section{RÉFÉRENCES}

13. Inui M, Saito A, Fleischer S. Purification of the ryanodine receptor and identification with fect structures of functional terminal cisternae of sarcoplasmic reticulum from fast skeletal muscle. J Biol Chem 1987 ; $262: 1740-7$

14. Costello B Chadwick C, Saito A Chu A, Maurer A, Fleischer S. Characterization of the junctional face membrane of terminal cisternae of sarcoplasmic reticulum. J Cell Biol 1986 ; 103 : 741-53.

15. MacLennan DH, Brandl CJ, Korizak B, Green NM. Amino-acid sequence of $\mathrm{Ca}^{2}+, \mathrm{Mg}^{2}+$ dependent ATPase from rabbit muscle sarcoplasmic reticulum deduced from its complementary DNA sequence. Nature 1985 ; 316 : 696-700.

16. Kawamoto RM, Brunschwig JP, Kim KC, Caswell AM. Isolation, characterization and localization of the spanning protein from skeletal muscle triade. J Cell Biol 1986 ; 103 : 1405-14.

17. Palade $\mathrm{P}$. Drug induced $\mathrm{Ca}^{2}+$ release from isolated sarcoplasmic reticulum. III. Biol Chem 1987 ; 262 : 6149-54.

18. Rousseau E, Meissner G. Single cardiac sarcoplasmic reticulum $\mathrm{Ca}^{2}+$ release channel : activation by caffeine. Am J Physiol $1989 ; 256: 328-33$

19. Sutko JL, Kenyon JL. Ryanodine modification of cardiac muscle responses to potassium-free solutions. Evidence for inhibition of sarcoplasmic reticulum $\mathrm{Ca}^{2}+$ release. J Gen Physiol 1983 ; 385 : 404.

20. Pessah IN, Francini AO, Scales DJ, Waterhouse AL, Casida JE. Calcium ryanodine receptor complex. Solubilization and partial characterization from skeletal muscle junctional sarcoplasmic reticulum. $J$ Biol Chem 1986; 261 : 8643-8.

21. Anderson $K$, Lai AF, Liu QY, Rousseau E, Erickson HP, Meissner G. Structural and functional characterization of the purified cardiac ryanodine receptor- $\mathrm{Ca}^{2}+$ release channel complex. J Biol Chem 1989 ; 264: 1329-35.

22. Smith JS, Imagawa $\mathrm{T}, \mathrm{Ma} J$, Fill $\mathrm{M}$ Campbell K, Coronado R. Purified ryanodine receptor from rabbit skeletal muscle in the calcium release channel of sarcoplasmic reticulum. J Gen Physiol 1988 ; 92 : 1-26.

23. Radon DP, Cefali DC, Mitchell RD, Seiler SM, Jones LR. High molecular weight proteins purified from cardiac junctional sarcoplasmic reticulum vesicles are ryanodine sensitive calcium channels. Circ Res 1990 ; 64 : 779-89.

24. Caswell AH, Brandt NR, Brunschwig JP, Purkerson S. Localization and partial characterization of the oligomeric disulfide-linked molecular weight 95000 protein (triadin) which bind the ryanodine and dihydropyridine receptors in skeletal muscle triadic vesicles. Biochemistry 1991; 30 : 7507-13
d'ATP (figure 4C). Le $\mathrm{Mg}^{2+}$ (figure $4 D)$, la calmoduline et le rouge de ruthénium - qui sont des bloqueurs connus du flux sortant rapide de $\mathrm{Ca}^{2+}$ - se sont révélés des inhibiteurs efficaces de cette activité électrique [7-9], alors que la caféine agit comme un agoniste partiel [9-18]. En revanche, les inhibiteurs classiques des canaux calciques des membranes de surface, telles les dihydropyridines et les phénylalkylamines, n'ont pas d'effet direct sur ce type de canal. De plus, l'activité de ce canal n'est pas stimulée par l'addition d'IP3 [8]. A faible concentration, la ryanodine modifie de façon pratiquement irréversible la conductance et le mode de fonctionnement des canaux unitaires du réticulum sarcoplasmique en maintenant

\section{A $0,1 \mu \mathrm{M} \mathrm{Ca}^{2+}$}
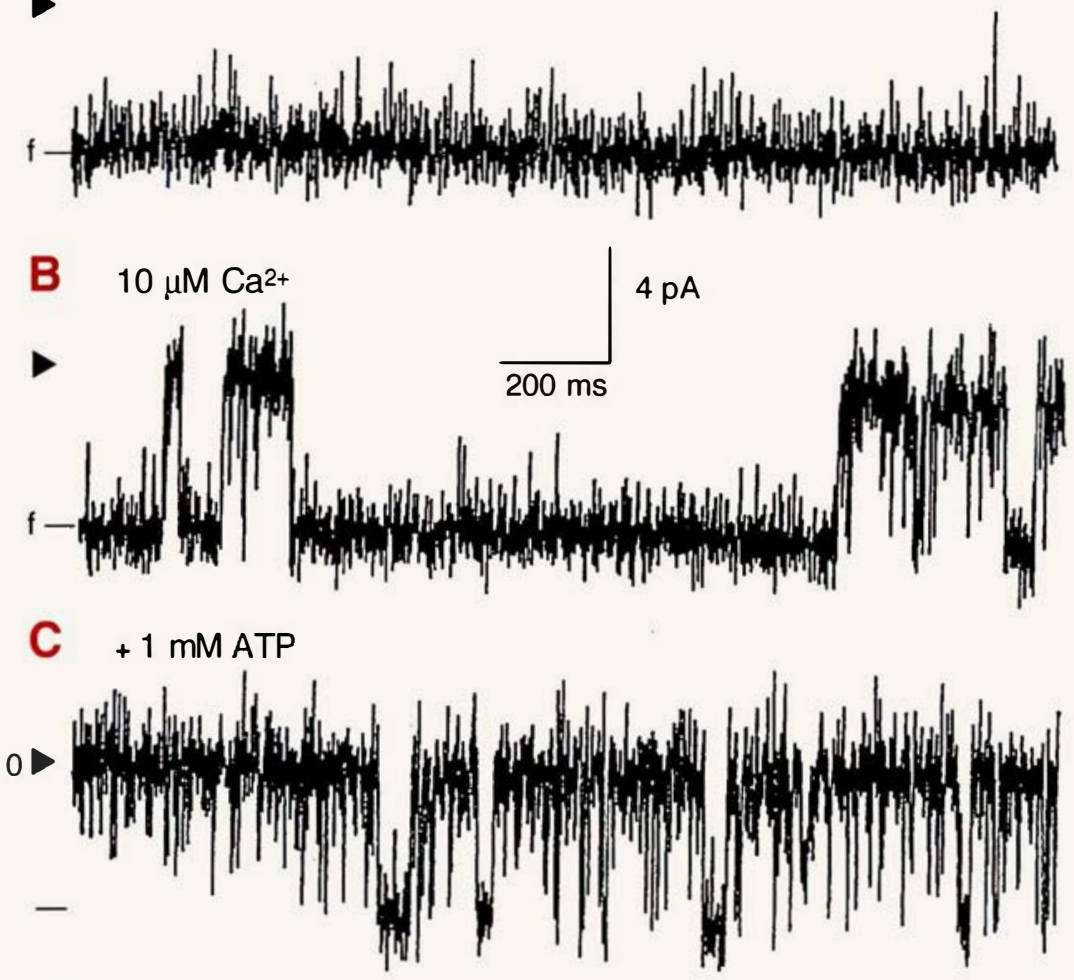

D $+2,5 \mathrm{mM} \mathrm{Mg}^{2+}$

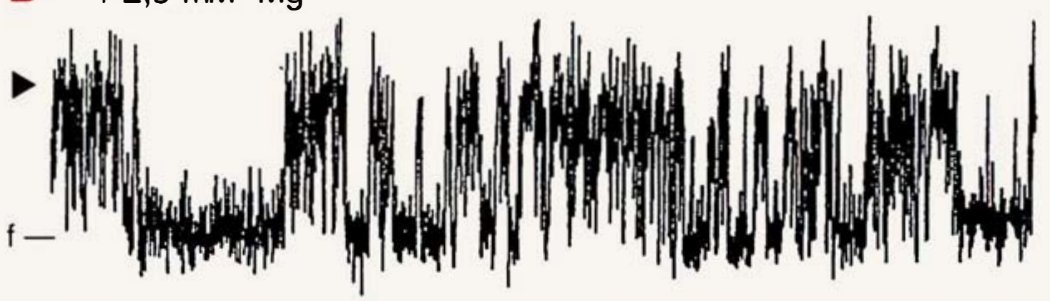

Figure 4. Enregistrements de l'activité du canal calcique du réticulum sarcoplasmique modulé par différents ligands. L'activité du canal est enregistrée en condition asymétrique après fusion d'une microvésicule dérivée du réticulum sarcoplasmique cardiaque dans une bicouche lipidique plane (voir figure 3). (A) En présence de $0,1 \mu \mathrm{M}$ de $\mathrm{Ca}^{2}+$ libre du côté cytoplasmique (cis), le canal est pratiquement toujours dans l'état fermé (f). (B) En présence de $10 \mu \mathrm{M}$ de $\mathrm{Ca}^{2+}$, la probabilité d'ouverture augmente. (C) Après ajout $d^{\prime} A T P(1 \mathrm{mM})$, le canal est maintenu dans son état ouvert (o). (D) L'addition de $\mathrm{Mg}^{2+}(2,5 \mathrm{mM})$ du côté cytoplasmique (cis) inhibe l'activité de ce canal en modifiant sa cinétique. 
ces derniers dans un état sousconducteur de longue durée [10]. Dans cet état sous-conducteur, le canal devient alors insensible à l'action de ses ligands physiologiques: $\mathrm{Ca}^{2+}$, ATP, $\mathrm{Mg}^{2+}$. Cet effet serait dû à l'interaction de la ryanodine avec un site de haute affinité dont l'existence a été confirmée par des études biochimiques [20]. A forte concentration (> $50 \mu \mathrm{M})$, l'interaction avec un site de basse affinité induit la fermeture du canal et une inhibition de la libération rapide de $\mathrm{Ca}^{2+}$ par le réticulum sarcoplasmique.

\section{Purification et reconstitution du récepteur de la ryanodine}

Le récepteur de la $\left[{ }^{3} \mathrm{H}\right]$-ryanodine fut solubilisé en présence de CHAPS (voir glossaire, p. 952) (1\%) et purifié sur gradient de sucrose [20]. Le complexe ainsi purifié a un coefficient de sédimentation très élevé, de l'ordre de $30 \mathrm{~S}$ [11]. Le pic de radioactivité est associé à un petit pic dc protéine qui, analysé sur gel d'électrophorèse, contient essentiellement une bande protéique de très haute masse moléculaire $(\mathrm{Mr}>400 \mathrm{~K})$ suggérant que le récepteur de la ryanodine est un énorme complexe oligomérique [11]. Observé en microscopie électronique à haute résolution, le récepteur de la ryanodine non dénaturé a les dimensions et l'aspect d'un trèfle à quatre feuilles similaire aux piliers (feet structure) qui relient le reticulum sarcoplasmique au système $T[11,14]$. Lorsque le complexe protéique, récupéré du gradient de sucrose, est incorporé dans des membranes artificielles en utilisant des conditions expérimentales similaires à celles décrites sur la figure 3, il est possible d'enregistrer l'activité électrique de canaux sélectifs des cations divalents [11, 21-23]. Néanmoins, en l'absence de $\mathrm{Ca}^{2+}$, cette structure protéique peut également conduire des cations monovalents : $\mathrm{Na}^{+}$ou $\mathrm{K}^{+}$[21-22]. Cette propriété a été mise à profit pour étudier la conductance au $\mathrm{Na}^{+}$de l'homotétramère, en fonction du $\mathrm{Ca}^{2+}$ côté cytoplasmique (cis). Plusieurs laboratoires ont démontré que des augmentations de $\mathrm{Ca}^{2+}$ (de 0,1 à $10 \mu \mathrm{M})$ du côté cytoplasmique du canal reconstitué favorisent son ouverture de façon similaire à ce qui a été observé pour le canal calcique du réticulum sarcoplasmique natif $[21,23]$. Une probabilité d'ouverture plus élevée est obtenue lorsque le complexe protéique reconstitué est activé en présence de $\mathrm{Ca}^{2+}$ et d'ATP. La caféine a également un effet agoniste sur le complexe protéique reconstitué [9]. En revanche, le $\mathrm{Mg}^{2+}(1 \mathrm{mM})$ et le rouge de ruthénium $(10 \mu \mathrm{M})$ inhibent complètement son activité. Quant à la ryanodine, elle modifie à la fois la conductance et la cinétique du canal purifié et reconstitué [22, 23] de façon identique à celle du canal natif [10]. Tous ces résultats tendent à prouver que le récepteur de la ryanodine des muscles striés est la structure protéique qui contrôle la libération rapide du $\mathrm{Ca}^{2+}$ à travers la membrane du réticulum sarcoplasmique lors du couplage excitation-contraction. Cette structure forme un canal dont l'activité est très dépendante des concen- trations de $\mathrm{Ca}^{2+}$, d'ATP et de $\mathrm{Mg}^{2+} \mathrm{du}$ côté cytoplasmique. Elle présente donc les caractéristiques d'un canal activé par ligands. Les canaux de ce type sont connus pour être peu dépendants du voltage. Toutefois, in situ, l'énorme complexe protéique que constitue le récepteur de la ryanodine pourrait être en relation avec des protéines du système $T$ qui, elles, seraient sensibles aux variations de champ électrique résultant du potentiel d'action [3]. Ainsi, au moins dans le muscle, le récepteur des dihydropyridines situé dans la membrane cellulaire des tubules transverses du système $T$ pourrait être présenté comme l'élément sensible au voltage impliqué dans les interactions moléculaires entre les composants du système $\mathrm{T}$ et ceux du réticulum sarcoplasmique (figure 5). De plus, une protéine de $95 \mathrm{kDa}$, appelée triadine, a été récemment isolée et localisée sur le feuillet interne de la membrane du système $\mathrm{T}$ [24]. Cette protéine se lie à la fois au récepteur de la ryanodine et au

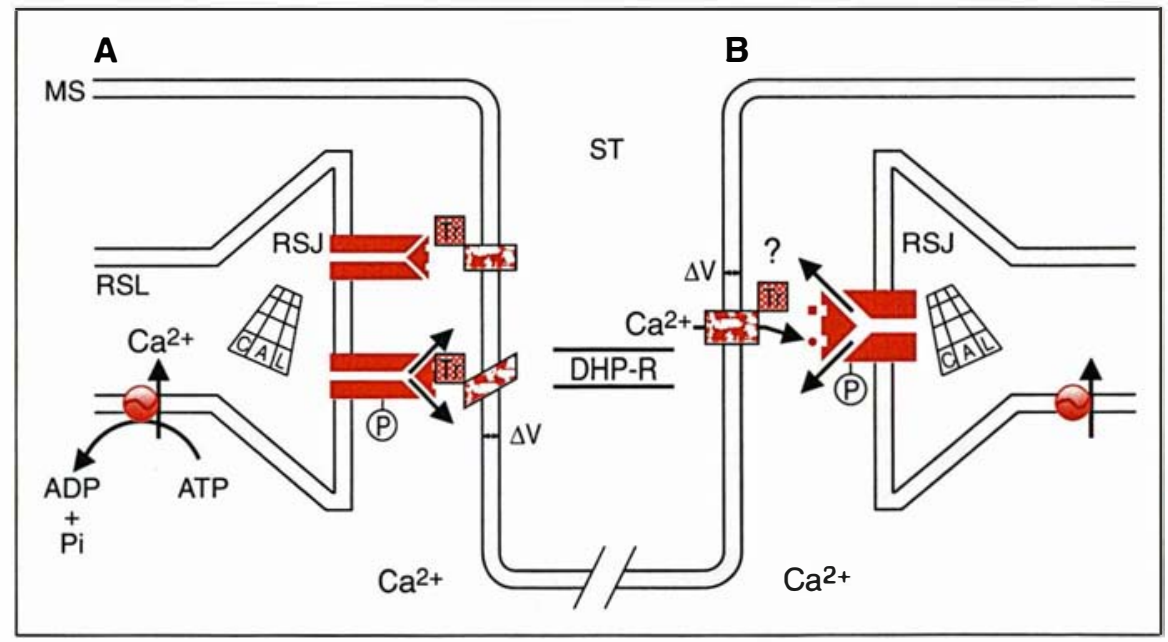

Figure 5. Localisation et topologie du canal calcique du réticulum sarcoplasmique. Ce canal comporte des sites d'activation au $\mathrm{Ca}^{2+}(\mathrm{O})$ et à I'ATP (प) du côté cytoplasmique, et établit vraisemblablement des interactions avec des composantes protéiques de la membrane du système tubulaire transverse : ST. MS : membrane de surface; RSL : réticulum sarcoplasmique longitudinal ; RSJ : réticulum sarcoplasmique jonctionnel ; DHP-R : récepteur aux dihydropyridines ; CAL : calséquestrine ; $\triangle V$ : variation de potentiel membranaire; Tr: Triadine; $P$ - : site de phosphorylation [37]. Deux modes de couplage sont postulés: (A) Au niveau du muscle squelettique, la dépolarisation de la membrane du ST induit un changement de conformation du DHP-R ldu fait des variations des champs électriques) qui serait communiqué au canal calcique du RS par l'intermédiaire de la triadine. (B) Au niveau du cœur, le DHP-R serait un canal calcique activé par la dépolarisation. Il permettrait l'entrée de $\mathrm{Ca}^{2+}$ et l'activation ainsi induite du canal calcique du réticulum sarcoplasmique. La présence de triadine est encore hypothétique au niveau du muscle cardiaque. 


\section{RÉFÉRENCES}

25. Hermann-Frank A, Darling E, Meissner G. Functional characterization of the $\mathrm{Ca}^{2+}$ gated $\mathrm{Ca}^{2+}$ release channel of vascular smooth muscle sarcoplasmic reticulum. Pflüg Arch 1991; 418 : 353-9.

26. Bourreau JP, Abela AP, Kwan CY, Daniel EE. Acetylcholine $\mathrm{Ca}^{2+}$ stores refilling directly involves a dihydropyridine sensitive channel in dog trachea. Am J Physiol 1991 ; 261 : C497-505.

27. Savaria D, Lanoué C, Cadieux A Rousseau E. Large conducting $\mathrm{K}^{+}$channel reconstituted from airway smooth muscle. Am J Physiol 1992 (sous presse).

28. McPherson PS, Campbell K. Solubilization and biochemical characterization of the high affinity $\left[{ }^{3} \mathrm{H}\right]$-ryanodine receptor from rabbit brain membrane. J Biol Chem 1990 ; 265 : 18454-60.

29. MacPherson PS, Kim YK, Valdivia $\mathrm{H}$ et al. The brain ryanodine receptor: a caffeine sensitive calcium channel. Neuron $1991 ; 7: 17-25$.

30. Takeshima M, Nishimura S, Matsumoto T, et al. Primary structure and expression from complementary DNA of skeletal muscle ryanodine receptor. Nature 1989 ; 338 : 439-45.

31. Otsu K, Willard HF, Khanna VK, Zorzato F, Green NM, Mac Lennan DH. Molecular cloning of cDNA encoding the $\mathrm{Ca}^{2}+$ release channel (ryanodine receptor) of rabbit cardiac muscle sarcoplasmic reticulum. J Biol Chem 1990 ; 265 : 13472-83.

32. Michelson JR, Ross JA, Reed BK, Louis CF. Enhanced $\mathrm{Ca}^{2+}$ induced calcium release by isolated sarcoplasmic reticulum vesicles from malignant hyperthermia susceptible pig muscle. Biochim Biophys Acta $1986 ; 862$ : 318-28.

33. Michelson JR, Gallant EM, Litterer LA, Johnson KM, Remyel WE, Louis CF. Abnormal sarcoplasmic reticulum ryanodine receptor in malignant hyperthermia. J Biol Chem 1988; 263 : 9310-5.

34. MacLennan DH, Duff C, Zorzato F, et al. Ryanodine receptor gene is a candidate for predisposition to malignant hyperthermia. Nature 1990 ; 343 : 559-61.

35. MacLennan DH, Phillips MS. Malignant hyperthermia. Science 1992 ; 256 : 789-94.

36. Sauvé R. Le patch clamp, une nouvelle façon de voir les canaux ioniques. médecine/sciences $1987 ; 3$ : 538-45.

37. Witcher DR, Kowacs R, Schulman H, Cefali D, Jones LR. Unique phosphorylation site on the cardiac ryanodine receptor regulates calcium channel activity. $J$ Biol Chem $1991 ; 266$ : 11144-52. récepteur des dihydropyridines alors que ces deux types de récepteurs ne présentent pas d'affinité l'un pour l'autre [24]. Ces résultats laissent entrevoir la possibilité que la triadine pourrait représenter l'un des rouages du couplage entre l'excitation et la contraction.

\section{Autre localisation du récepteur de la ryanodine}

Un autre site de liaison spécifique de haute affinité pour la $\left[{ }^{3} \mathrm{H}\right]$-ryanodine a été détccté dans des fractions microsomales isolées de muscles lisses vasculaire [25] et respiratoire [26, 27] ainsi que dans des membranes isolées de cerveau de mammifere [28]. Bien que dans ces différents tissus le rôle exact de ce récepteur ne soit pas encore connu, il est possible, à la lumière de données pharmacologiques et fonctionnelles récentes, de postuler qu'il puisse être également impliqué dans la libération intracellulaire du $\mathrm{Ca}^{2+}[25-29]$.

\section{Informations obtenues de la génétique moléculaire}

La séquence en acides aminés du polypeptide constituant le récepteur de la ryanodine a été déduite après clonage et analyse des ADN complémentaires dans le cœur et le muscle. Les récepteurs des muscles squelettique et cardiaque sont respectivement composés de 5037 [30] et 4969 [31] acides aminés avec un pourcentage d'homologie de $66 \%$. Par ailleurs, les similitudes entre les profils d'hydropathie suggèrent que les deux isof ormes ont globalement la même topologie. Si plusieurs sites de liaison potentielle pour la calmoduline ont été observés, il est cependant surprenant qu'aucun domaine de haute affinité pour le $\mathrm{Ca}^{2+}$ et pour l'ATP n'ait pu être déduit de la séquence primaire [31]. En revanche, un consensus s'est établi sur le fait que les segments transmembranaires qui forment le canal dans la membrane du réticulum sarcoplasmique soient localisés dans la région C-terminale.

\section{De la biologie moléculaire} au diagnostic médical

Une affection rare, mais létale, est reliée à un défaut du contrôle de la libération du $\mathrm{Ca}^{2}+$ au niveau des muscles squelettiques. Il s'agit de l'hyperthermie maligne, caractérisée par une hypersensibilité aux anesthésiques volatils tels que l'halothane [32]. Les patients développant ce syndrome réagissent de façon spontanée ou retardée aux anesthésiques par des contractures intenses, une élévation soudaine, importante et irréversible de leur température corporelle $\left(37\right.$ à $42^{\circ} \mathrm{C}$ ) qui peut conduire au décès. Ces patients doivent être rapidement traités au dendrolène, un inhibiteur de la libération du $\mathrm{Ca}^{2+}$. Cette physiopathologie a été étudiée en détail chez le porc [32, 33]. Un mécanisme d'induction complet a récemment été décrit (figure 3 dans [34]). Il implique une sensibilité au $\mathrm{Ca}^{2+}$ accrue pour différents phénomènes intracellulaires et entraînerait une cascade d'événements tels que l'augmentation de la libération de $\mathrm{Ca}^{2+}$ par le réticulum sarcoplasmique, de la contraction, de la consommation d'oxygène et de la production de chaleur. Son dépistage chez l'homme, ou chez les porcins d'élevage, reste subordonné à des tests fastidieux, plus ou moins fiables (test de contracture à la caféine et à l'halothane), réalisés sur des biopsies musculaires. Chez le porc, cette physiopathologie est directement reliée à un défaut du mode de régulation du canal calcique du réticulum sarcoplasmique : forte sensibilité au $\mathrm{Ca}^{2+}$ et à l'halothane, affinité augmentée pour la ryanodine et défaut d'inactivation [32]. Ces résultats suggèrent un défaut génétique au niveau de l'expression de la protéine formant le canal calcique du réticulum sarcoplasmique, alias le réccpteur de la ryanodine. En revanche, chez l'homme, différentes formes de réponse à l'halothane, lors des tests de contracture, suggèrent l'existence de plusieurs formes d'hyperthermie maligne qui pourraient être reliées à des défauts génétiques impliquant diverses protéines sensibles au $\mathrm{Ca}^{2+}[35]$; d'où la nécessité de produire un kit de diagnostic très fiable permettant de détecter la (ou les) différente(s) mutation(s). Les progrès actuels réalisés en génétique moléculaire laissent penser qu'il sera bientôt possible d'identifier les sujets atteints de ces mutations récessives dans les familles à risque 
qui sont répertoriées en Europe et au Canada. Le phénotype silencieux et invisible sera démasqué en utilisant la technique de PCR et des sondes biologiques dirigées contre la région q13.1 de leur chromosome 19 qui code pour la protéine défectueuse [34, 35].

\section{Conclusion}

L'identification fonctionnelle et structurale du complexe protéique qui forme le canal calcique du réticulum sarcoplasmique des muscles cardiaque et squelettique constitue une contribution majeure dans la compréhension des mécanismes qui contrôlent la libération rapide $\mathrm{de} \mathrm{Ca}^{2+}$ lors $\mathrm{du}$ couplage entre l'excitation et la contraction. Ce canal qui fut purifié et reconstitué sous la forme du récepteur de la ryanodine, a des propriétés biochimiques, biophysiques et pharmacologiques qui le distinguent des autres types de canaux calciques, que ce soient ceux de type $\mathrm{L}, \mathrm{T}, \mathrm{N}$ des membranes de surface ou bien encore celui du réticulum endoplasmique identifié comme le récepteur d'IP3. Des publications récentes ont démontré que le récepteur de la ryanodine est également présent dans différents tissus nerveux, ce qui a renforcé l'intérêt des recherches sur le rôle et la fonction des différentes isoformes exprimées par les cellules non musculaires

\section{Summary}

Functional identification of the $\mathrm{Ca}^{2+}$ release channel from sarcoplasmic reticulum

Striated muscles contract following the rapid and transient raise of intracellular free $\mathrm{Ca}^{2+}$. Most of this $\mathrm{Ca}^{2}+$ is believed to be released from the sarcoplasmic reticulum (SR). In order to study the calcium conductances of the SR membrane, microsomal fractions were isolated by differential and sucrose gradient centrifugations and fused into planar lipid bilayers (PLB) made of phospholipids. Using an asymmetrical $\mathrm{Ca}^{2+}$ buffer system the activity of a large $\mathrm{Ca}^{2+}$ conducting channel was recorded $(100 \mathrm{pS}$ in $50 \mathrm{mM}$ $\mathrm{Ca}^{2+}$ trans). This channel was activated by $\mathrm{Ca}^{2+}$ while its $\mathrm{Ca}^{2+}$-sensitivity was potentiated by ATP. However $\mathrm{Mg}^{2+}$ and calmodulin modulated its gating behavior. A $\mathrm{Ca}^{2+}$ releasing drug such as caffeine increased its open probability. Ryanodine has been shown to modify the conducting and the gating behaviour of this channel. All these features are characteristics of the native $\mathrm{SR} \mathrm{Ca}^{2+}$ release channel when it is studied using isotopic ${ }^{45} \mathrm{Ca}^{2+}$ flux or spectroscopic measurements. The ryanodine receptor has been identified and purified to homogeneity using sucrose gradient and affinity chromatography. When reconstituted into PLB, the $30 \mathrm{~S}$ protein complex forms a divalent cation selective channel. This multimeric complex displays all the regulatory sites of the native $\mathrm{SR} \mathrm{Ca}^{2+}$ release channel. In conclusion, the reconstitution experiments have allowed biochemical and functional identification of the $\mathrm{Ca}^{2+}$ release channel that regulates the ionic flux across the SR membrane. Recently, it was shown that the malignant hyperthermia syndrome was related to a mutation of the gene encoding the protein supporting this channel activity.

\section{Remerciements}

Ce travail a bénéficié des programmes de subvention de recherche du Conseil de recherche médical du Canada (CRMC), de la Fondation du Québec des Maladies du cœur (FQMC) et du Fonds de recherche en santé du Québec (FRSQ). Éric Rousseau est chercheur-boursier et Hélène Chabot est boursière de la Fondation des Maladies du cœur au Canada. Les auteurs remercient les Drs Marcel-Daniel Payet et Nicole Gallo-Payet pour leurs commentaires judicieux ainsi que $\mathrm{M}$ mes Catherine Beaudry et Christiane Ducharme pour l'acquisition des résultats et la mise en forme du manuscrit. 